Alternating Gradient Synchrotron Department

Relativistic Heavy Ion Collider Project

BROOKHAVEN NATIONAL LABORATORY

Upton, New York 11973

Spin Note

AGS/RHIC/SN No. 63

\title{
Expressions For The Magnetic Flux \\ Enclosed By Rotating Coils \\ In Helical Dipoles
}

\author{
Toshiharu Tominaka \\ AGS Department, Brookhaven National Laboratory, NY 11973
}

September 22, 1997 


\section{EXPRESSIONS FOR THE MAGNETIC FLUX \\ ENCLOSED BY ROTATING COILS \\ IN HELICAL DIPOLES}

T. Tominaka (RIKEN, Japan)

September 12, 1997

\section{INTRODUCTION}

The expressions for the magnetic flux enclosed by various coils in helical dipoles are derived for the analysis of the multipole contents.[1,2,3]

\section{ANALYTICAL EXPRESSION FOR THE MAGNETIC FIELD OF HELICAL DIPOLES}

\subsection{European Definition}

The interior magnetic field of helical dipole coil with an infinite length can be described as follows, [4]

$$
\left\{\begin{array}{c}
B_{r}(r, \theta, z)=B_{\text {ref }}(k) r_{0} \sum_{n=1}^{\infty} n !\left[\frac{2}{n k r_{0}}\right]^{n} k I_{n}^{\prime}(n k r)\left\{-a_{n}(k) \cos (n(\theta-k z))+b_{n}(k) \sin (n(\theta-k z))\right\} \\
B_{\theta}(r, \theta, z)=B_{\text {ref }}(k) r_{0} \sum_{n=1}^{\infty} n !\left[\frac{2}{n k r_{0}}\right]^{n} \frac{I_{n}(n k r)}{r}\left\{a_{n}(k) \sin (n(\theta-k z))+b_{n}(k) \cos (n(\theta-k z))\right\}=-\frac{B_{z}(r, \theta, z)}{k r} \\
B_{z}(r, \theta, z)=B_{r e r}(k) r_{0} \sum_{n=1}^{\infty}(-k) n !\left[\frac{2}{n k r_{0}}\right]^{n} I_{n}(n k r)\left\{a_{n}(k) \sin (n(\theta-k z))+b_{n}(k) \cos (n(\theta-k z))\right\}
\end{array}\right.
$$

\subsection{American Definition}

With the following replacement for Eq.(1), the expression for the American definition can be obtained, $[2,3]$

$\left\{\begin{array}{l}B_{\text {ref }}(k)=B_{0} \\ a_{n}(k)=-\tilde{a}_{n-1} \\ b_{n}(k)=\tilde{b}_{n-1},\end{array}\right.$

resulting in the following expression,

$$
\left\{\begin{array}{c}
B_{n}(r, \theta, z)=B_{0} r_{0} \sum_{n=0}^{\infty}(n+1) !\left[\frac{2}{(n+1) k r_{0}}\right]^{(n+1)} k \dot{I}_{n+1}((n+1) k r)\left\{\tilde{a}_{n} \cos ((n+1)(\theta-k z))+\tilde{b}_{n} \sin ((n+1)(\theta-k z))\right\} \\
B_{\theta}(r, \theta, z)=-\frac{B_{z}(r, \theta, z)}{k r} \\
\left.B_{z}(r, \theta, z)=\left.B_{0} r_{0} \sum_{n=0}^{\infty}(-k)(n+1) !\left[\frac{2}{(n+1) k r_{0}}\right]^{(n+1)} I_{n+1}((n+1) k r)\right|_{i} ^{1} \tilde{a}_{n} \sin (n(\theta-k z))+\tilde{b}_{n} \cos (n(\theta-k z))\right\}
\end{array}\right.
$$

Eqs.(1) and (3) are equivalent through the relation between the multipole coefficients. In this article, Eq.(1) is utilized for the expressions for the magnetic flux enclosed by various coils in helical dipoles. 


\section{ANALYTICAL EXPRESSIONS FOR THE MAGNETIC FLUX ENCLOSED BY VARIOUS COILS IN HELICAL DIPOLES}

\subsection{Radial Coil}

3.1 .1 Coil with the radius, $\Delta r=r_{2}-r_{1}$ and the length, $\Delta z=z_{2}-z_{1}$

The angular magnetic flux, $\Phi_{\theta}(\theta)$, at the angle $\theta$, enclosed by the general radial coil with the radius, $\Delta r$ $=r_{2}-r_{1}$ and the length, $\Delta z=z_{2}-z_{1}$ in the helical dipole can be expressed as follows,

$$
\begin{gathered}
\Phi_{\theta}(\theta)=\int_{z_{1}}^{z_{2}} \int_{r_{1}}^{r_{2}} B_{\theta}(r, \theta, z) d r d z \\
=B_{\text {ref }}(k) r_{0} \sum_{n=1}^{\infty} n !\left[\frac{2}{n k r_{0}}\right]^{n} \int_{r_{1}}^{r_{2}} \frac{I_{n}(n k r)}{r} d r x \\
\int_{z_{1}}^{z_{2}}\left\{a_{n}(k) \sin (n(\theta-k z))+b_{n}(k) \cos (n(\theta-k z))\right\}_{t},
\end{gathered}
$$

resulting in the following expression,

$$
\begin{gathered}
\Phi_{\theta}(\theta)=B_{\text {ref }}(k) r_{0} \sum_{n=1}^{\infty} n !\left[\frac{2}{n k r_{0}}\right]^{n} \int_{r 1}^{r} \frac{n_{n}(n k r)}{r} d r \times \\
\left.+\sin n \theta\left\{+\frac{2 a_{n}(k)}{n k} \cos \frac{n k\left(z_{2}+z_{1}\right)}{2} \sin \frac{n k\left(z_{2}-z_{1}\right)}{2}+\frac{2 b_{n}(k)}{n k} \sin \frac{n k\left(z_{2}+z_{1}\right)}{2} \sin \frac{n k\left(z_{2}-z_{1}\right)}{2}\right\}\right] .
\end{gathered}
$$

3.1.2 Coil with the radius, $\Delta r=r_{g}$ and the length, $\Delta z$

The angular magnetic flux, $\Phi_{\theta}(\theta)$, at the angle $\theta$, enclosed by the radial coil with $r_{2}=r_{c}, r_{1}=0$ and $z_{1}=-$ $\Delta z / 2$ and $z_{2}=\Delta z / 2$, as shown in Fig.1, in the helical dipole can be expressed as follows,

$$
\begin{gathered}
\Phi_{\theta}(\theta)=\int_{-\Delta z / 2}^{\Delta z / 2} \int_{0}^{r_{c}} B_{\theta}(r, \theta, z) d r d z \\
=B_{\text {ref }}(k) r_{0} \sum_{n=1}^{\infty} n !\left[\frac{2}{n k r_{0}}\right]^{n} \int_{0}^{r_{c}} \frac{I_{n}(n k r)}{r} d r \times \\
\frac{2}{n k} \sin \left(n k \frac{\Delta z}{2}\right)\left\{+b_{n}(k) \cos n \theta+a_{n}(k) \sin n \theta\right\} .
\end{gathered}
$$

3.1.3 Coil with the radius, $r_{8,}$, the spanned angle, $\Delta \theta=\pi$ and the length, $\Delta z$

The angular magnetic flux, $\Phi_{\theta}(\theta)$, at the angle $\theta$, enclosed by the radial coil with $r_{2}=r_{c}, r_{1}=0$ and $z_{1}=-$ $\Delta z / 2, z_{2}=\Delta z / 2$, and the spanned angle, $\Delta \theta=\pi$, as shown in Fig.2, in the helical dipole can also be expressed as follows,

$$
\begin{gathered}
\Phi_{\theta}(\theta)=\int_{-\Delta z / 2}^{\Delta z / 2} \int_{0}^{r_{c}} B_{\theta}(r, \theta, z) d r d z+\int_{-\Delta z / 2}^{\Delta z / 2} \int_{0}^{s_{c}}-B_{\theta}(r, \theta+\pi, z) d r d z \\
=B_{r e f}(k) r_{0} \sum_{n=1}^{\infty} n !\left[\frac{2}{n k r_{0}}\right]^{n} \int_{0}^{s_{c}} \frac{I_{n}(n k r)}{r} d r x \\
\left\{1-(-1)^{n}\right\} \frac{2}{n k} \sin \left(n k \frac{\Delta z}{2}\right)\left\{+b_{n}(k) \cos n \theta+a_{n}(k) \sin n \theta\right\}
\end{gathered}
$$




\subsection{Tangential-like Coil}

3.2.1 Coil with the radius, $r_{c^{2}}$ the spanned angle, $\Delta \theta=\theta_{2}-\theta_{1}$ and the length, $\Delta z$

The radial magnetic flux, $\Phi_{r}(\theta)$, at the angle $\theta$, enclosed by the general tangential coil with the radius, $r_{c}$, the spanned angle, $\Delta \theta=\theta_{2}-\theta_{1}$ and the length, $z_{1}=-\Delta z / 2$ and $z_{2}=\Delta z / 2$ in the helical dipole can be expressed as follows,

$$
\begin{aligned}
& \Phi_{\mathrm{r}}(\theta)=\int_{-\Delta z / 2}^{\Delta z / 2} \int_{\theta+\theta_{1}}^{\theta+\theta_{2}} B_{\mathrm{r}}\left(r_{\mathrm{c}}, \theta, z\right) r_{\mathrm{c}} d \theta d z \\
& =B_{\text {ref }}(k) r_{0} \sum_{n=1}^{\infty} n !\left[\frac{2}{n k r_{0}}\right]^{n} k I_{n}^{\prime}(n k r) \times r \times \\
& \int_{-\Delta z / 2}^{\Delta z / 2} \int_{\theta+\theta 1}^{\theta+\theta_{2}}\left\{-a_{n}(k) \cos (n(\theta-k z))+b_{n}(k) \sin (n(\theta-k z))\right\} d \theta d z
\end{aligned}
$$

3.2.2 Coil with the radius, $I_{8}$, the spanned angle, $\Delta \theta=\pi$ and the length. $\Delta z$ (Dipole Winding)

The radial magnetic flux, $\Phi_{r}(\theta)$, at the angle $\theta$, enclosed by the dipole winding with the radius, $r_{c}, z_{1}=-$ $\Delta z / 2, z_{2}=\Delta z / 2$, and the spanned angle, $\Delta \theta=\pi$, as shown in Fig. 3 in the helical dipole can be expressed as follows,

$$
\begin{gathered}
\Phi_{\mathrm{r}}(\theta)=\int_{-\Delta z / 2}^{\Delta z / 2} \int_{\theta}^{\theta+\pi} B_{\mathrm{r}}\left(r_{c}, \theta, z\right) r_{c} d \theta d z \\
=B_{\mathrm{ref}}(k) r_{0} \sum_{n=1}^{\infty} n !\left[\frac{2}{n k r_{0}}\right]^{n}\left\{k r_{c} I_{n-1}\left(n k r_{c}\right)-I_{n}\left(n k r_{c}\right)\right\} \times \\
\int_{-\Delta z / 2}^{\Delta z / 2} \int_{\theta}^{\theta+\pi}\left\{-a_{n}(k) \cos (n(\theta-k z))+b_{n}(k) \sin (n(\theta-k z))\right\} d \theta d z
\end{gathered}
$$

resulting in the following expression,

$$
\begin{gathered}
\Phi_{r}(\theta)=B_{\text {ref }}(k) r_{0} \sum_{n=1}^{\infty} n !\left[\frac{2}{n k r_{0}}\right]^{n}\left\{k r_{c} I_{n-1}\left(n k r_{c}\right)-I_{n}\left(n k r_{c}\right)\right\} \times \\
\left\{1-(-1)^{n}\right\} \frac{2}{k n^{2}} \sin \left(n k \frac{\Delta z}{2}\right)\left\{b_{n}(k) \cos n \theta+a_{n}(k) \sin n \theta\right\} .
\end{gathered}
$$

Then, the factor of the $n$ dependence for $\Phi_{r}(\theta), S(n)$, can be introduced as follows,

$$
S(n)=n !\left[\frac{2}{n k r_{0}}\right]^{n}\left\{k r_{c} I_{n-1}\left(n k r_{c}\right)-I_{n}\left(n k r_{c}\right)\right\}\left\{1-(-1)^{n}\right\} \frac{2}{k n^{2}} \sin \left(n k \frac{\Delta z}{2}\right) \text {. }
$$

As a result, the relative sensitivity of the $2 \mathrm{n}$-pole magnetic flux for the dipole flux, $\dot{\mathrm{F}}_{\mathrm{s}}$, can be expressed as follows,

$$
F_{s}=\left|\frac{S(n)}{S(1)}\right| \text {. }
$$

\subsubsection{Coil with the radius. $r_{s}$ the spanned angle. $\Delta \theta=\pi / 12$ and the length. $\Delta z$ (Tangential Winding)} The radial magnetic flux, $\Phi_{\mathrm{r}}(\theta)$, at the angle $\theta$, enclosed by the tangential winding with the radius, $\mathrm{r}_{\mathrm{c}}, \mathrm{z}_{1}$ $=-\Delta z / 2, z_{2}=\Delta z / 2$, and the spanned angle, $\Delta \theta=\pi / 12$, as shown in Fig.4 in the helical dipole can be expressed as follows, 


$$
\begin{gathered}
\Phi_{\mathrm{r}}(\theta)=\int_{-\Delta z / 2}^{\Delta z / 2} \int_{\theta+11 \pi / 4}^{\theta+13 \pi / 24} B_{\mathrm{r}}\left(\mathrm{r}_{\mathrm{c}}, \theta, \mathrm{z}\right) \mathrm{r}_{\mathrm{c}} \mathrm{d} \theta \mathrm{dz} \\
=\mathrm{B}_{\mathrm{ref}}(\mathrm{k}) \mathrm{r}_{0} \sum_{\mathrm{n}=1}^{\infty} \mathrm{n} !\left[\frac{2}{\mathrm{nk} \mathrm{r}_{0}}\right]^{\mathrm{n}}\left\{\mathrm{k} \mathrm{r}_{\mathrm{c}} \mathrm{I}_{\mathrm{n}-1}\left(\mathrm{nk} \mathrm{r}_{\mathrm{c}}\right)-\mathrm{I}_{\mathrm{n}}\left(\mathrm{nk} \mathrm{r}_{\mathrm{c}}\right)\right\} \times \\
\int_{-\Delta z / 2}^{\Delta z / 2} \int_{\theta+11 \pi / 24}^{\theta+13 \pi / 24}\left\{-\mathrm{a}_{\mathrm{n}}(\mathrm{k}) \cos (\mathrm{n}(\theta-\mathrm{kz}))+b_{\mathrm{n}}(\mathrm{k}) \sin (\mathrm{n}(\theta-\mathrm{kz}))\right\} \mathrm{d} \theta \mathrm{dz},
\end{gathered}
$$

resulting in the following expression,

$$
\begin{gathered}
\Phi_{r}(\theta)=B_{\text {ref }}(k) r_{0} \sum_{n=1}^{\infty} n !\left[\frac{2}{n k r_{0}}\right]^{n}\left\{k r_{c} I_{n-1}\left(n k r_{c}\right)-I_{n}\left(n k r_{c}\right)\right\} \times \\
\sin \left(\frac{n \pi}{24}\right) \frac{4}{k n^{2}} \sin \left(n k \frac{\Delta z}{2}\right)\left\{b_{n}(k) \sin \left(n \theta+\frac{n \pi}{2}\right)-a_{n}(k) \cos \left(n \theta+\frac{n \pi}{2}\right)\right\} .
\end{gathered}
$$

Then, the factor of the $n$ dependence for $\Phi_{r}(\theta), S(n)$, can also be expressed as follows,

$$
S(n)=n !\left[\frac{2}{n k r_{0}}\right]^{n}\left\{k r_{c} I_{n-1}\left(n k r_{c}\right)-I_{n}\left(n k r_{c}\right)\right\} \sin \left(\frac{n \pi}{24}\right) \frac{4}{k^{2}} \sin \left(n k \frac{\Delta z}{2}\right)
$$

3.2.4 Coil with the radius, $r_{\alpha^{2}}$ the spanned angle, $\Delta \theta=\pi / 2 \times 2$ and the length, $\Delta z$ CQuadrupole

\section{Winding)}

The radial magnetic flux, $\Phi_{\mathrm{r}}(\theta)$, at the angle $\theta$, enclosed by the quadrupole winding with the radius, $\mathrm{r}_{\mathrm{c}}$, $z_{1}=-\Delta z / 2, z_{2}=\Delta z / 2$, and the spanned angle, $\Delta \theta=\pi / 2 \times 2$, as shown in Fig.5 in the helical dipole can be expressed as follows,

$$
\begin{aligned}
& \Phi_{\mathrm{r}}(\theta)=\int_{-\Delta z / 2}^{\Delta z / 2} \int_{\theta+\pi / 4}^{\theta+3 \pi / 4} B_{\mathrm{r}}\left(\mathrm{r}_{\mathrm{c}}, \theta, z\right) \mathrm{r}_{\mathrm{c}} d \theta d z+\int_{-\Delta z / 2}^{\Delta z / 2} \int_{\theta+5 \pi / 4}^{\theta+7 \pi / 4} B_{\mathrm{r}}\left(\mathrm{r}_{\mathrm{c}}, \theta, z\right) \mathrm{r}_{\mathrm{c}} d \theta d z \\
& \Phi_{r}(\theta)=B_{\text {ref }}(k) r_{0} \sum_{n=1}^{\infty} n !\left[\frac{2}{n k r_{0}}\right]^{n}\left\{k r_{c} I_{n-1}\left(n k r_{c}\right)-I_{n}\left(n k r_{c}\right)\right\} \times \\
& \left\{\int_{-\Delta z / 2}^{\Delta z / 2} \int_{\theta+\pi / 4}^{\theta+3 \pi / 4}\left\{-a_{n}(k) \cos (n(\theta-k z))+b_{n}(k) \sin (n(\theta-k z))\right\} d \theta d z+\right. \\
& \left.\int_{-\Delta z / 2}^{\Delta z / 2} \int_{\theta+5 \pi / 4}^{\theta+7 \pi / 4}\left\{-a_{n}(k) \cos (n(\theta-k z))+b_{n}(k) \sin (n(\theta-k z))\right\} d \theta d z\right\} \text {. } \\
& \Phi_{r}(\theta)=B_{r e f}(k) r_{0} \sum_{n=1}^{\infty} n !\left[\frac{2}{n k r_{0}}\right]^{n}\left\{k r_{c} I_{n-1}\left(n k r_{c}\right)-I_{n}\left(n k r_{c}\right)\right\} \times \\
& \left(\sin \left(\frac{\mathrm{n} \pi}{4}\right) \frac{4}{\mathrm{k} \mathrm{n}^{2}} \sin \left(\mathrm{nk} \frac{\Delta \mathrm{z}}{2}\right)\left\{\mathrm{b}_{\mathrm{n}}(\mathrm{k}) \sin \left(\mathrm{n} \theta+\frac{\mathrm{n} \pi}{2}\right)-\mathrm{a}_{\mathrm{n}}(\mathrm{k}) \cos \left(\mathrm{n} \theta+\frac{\mathrm{n} \pi}{2}\right)\right\}+\right. \\
& \left.\sin \left(\frac{\mathrm{n} \pi}{4}\right) \frac{4}{\mathrm{kn}^{2}} \sin \left(\mathrm{nk} \frac{\Delta \mathrm{z}}{2}\right)\left\{b_{n}(\mathrm{k}) \sin \left(\mathrm{n} \theta+\frac{3 \mathrm{n} \pi}{2}\right)-\mathrm{a}_{\mathrm{n}}(\mathrm{k}) \cos \left(\mathrm{n} \theta+\frac{3 \mathrm{n} \pi}{2}\right)\right\}\right) \text {. }
\end{aligned}
$$

As a result, the radial magnetic flux, $\Phi_{\mathrm{r}}(\theta)$, can be expressed as follows,

$$
\begin{gathered}
\Phi_{r}(\theta)=B_{r e f}(k) r_{0} \sum_{n=1}^{\infty} n !\left[\frac{2}{n k r_{0}}\right]^{n}\left\{k r_{c} I_{n-1}\left(n k r_{c}\right)-I_{n}\left(n k r_{c}\right)\right\} \times \\
(-1)^{n} \cos \left(\frac{n \pi}{2}\right) \sin \left(\frac{n \pi}{4}\right) \frac{8}{k n^{2}} \sin \left(n k \frac{\Delta z}{2}\right)\left\{b_{n}(k) \sin n \theta-a_{n}(k) \cos n \theta\right\}
\end{gathered}
$$


Then, the factor of the $\mathrm{n}$ dependence for $\Phi_{\mathrm{r}}(\theta), \mathrm{S}(\mathrm{n})$, can also be expressed,

$S(n)=n !\left[\frac{2}{n k r_{0}}\right]^{n}\left\{k r_{c} I_{n-1}\left(n k r_{c}\right)-I_{n}\left(n k r_{c}\right)\right\}(-1)^{n} \cos \left(\frac{n \pi}{2}\right) \sin \left(\frac{n \pi}{4}\right) \frac{8}{k n^{2}} \sin \left(n k \frac{\Delta z}{2}\right)$.

For $n=1$, especially, the following quantity, $S(1)$ derived by the removal of the factor, $\cos (n \pi / 2)$, form the Eq.(20) can be introduced in order to avoid $S(1)=0$,

$S(1)=\left[\frac{2}{n k r_{0}}\right]\left\{k r_{c} I_{0}\left(k r_{c}\right)-I_{1}\left(k r_{c}\right)\right\}(-1) \sin \left(\frac{\pi}{4}\right) \frac{8}{k} \sin \left(k \frac{\Delta z}{2}\right)$

\subsection{Comparison of the Relative Sensitivity among Different Windings}

Due to the existence of axial field, $B_{z}$ in helical dipoles, the enclosed magnetic fluxes of the radial coil shown in Fig. 2 and the dipole winding with the spanned angle, $\pi$, shown in Fig. 3 are different, whereas those are same in the $2 \mathrm{D}$ dipole. The relative sensitivity, $\mathrm{F}_{\mathrm{s}}$, among dipole, tangential, and quadrupole windings of the tangential-like coil are compared for the following values, in Table 1 and Fig.6,

reference radius : $r_{0}=31 \mathrm{~mm}$,

twist pitch of helical dipole : $\mathrm{k}=2 \pi / 2.4(\mathrm{rad} / \mathrm{m})=150.0(\mathrm{deg} / \mathrm{m})$,

radius of rotating coil : $r_{\mathrm{c}}=27.4 \mathrm{~mm}$, and

length of rotating coil : $\Delta z=9 \mathrm{in} .=228.6 \mathrm{~mm}$.

These values are typical ones for each winding of the rotating coil utilized for the magnetic field measurement of the prototype helical dipole magnet.

\section{CONCLUSION}

The expression for the magnetic flux enclosed in various coils are obtained, emphasizing the rotating coils utilized for the magnetic measurement of the prototype helical dipole.

\section{ACKNOWLEDGMENTS}

The author is indebted for helpful discussions and comments to A. Jain, R. Thomas, E. Willen, M. Okamura, and T. Katayama.

\section{REFERENCES}

1) A. Jain, Private communications.

2) W. Fisher, "On Measurements of Helical Magnetic Fields Using Devices for Straight Magnets", AGS/RHIC/SN No.32, July 30, (1996).

3) W. Fisher and M. Okamura, "Parameterization and Measurements of Helical Magnetic Fields", Proc. 1997 Part. Accel. Conf., Vancouver, to be published.

4) T. Tominaka, M. Okamura and T. Katayama, "Analytical Field Calculation of Helical Dipole Magnets for RHIC", Proc. 1997 Part. Accel. Conf., Vancouver, to be published. 


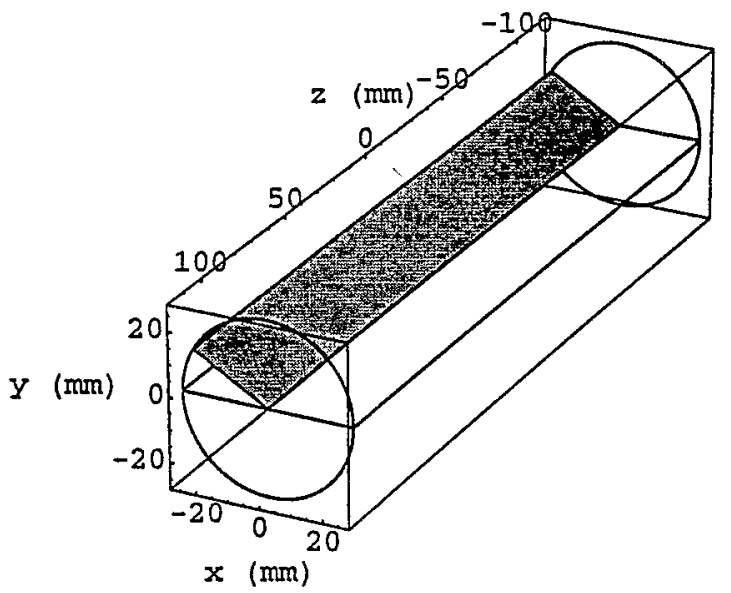

Fig.1 Radial coil \#1.

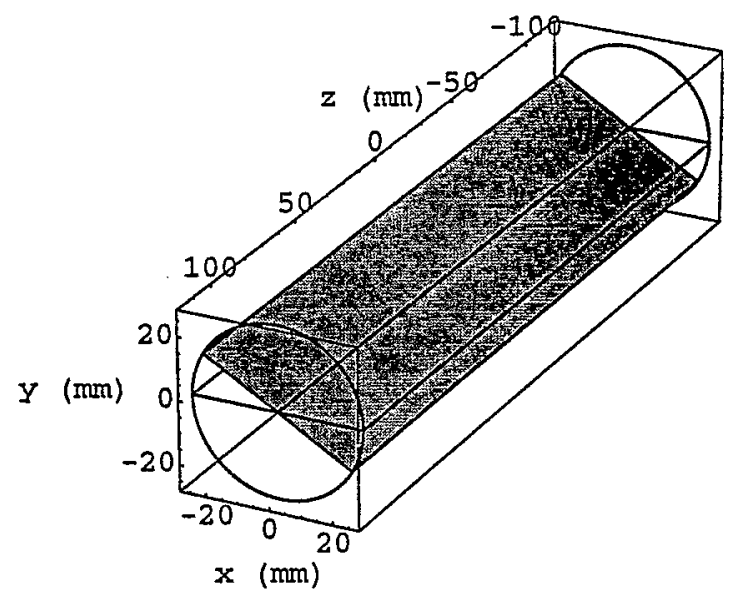

Fig.2 Radial coil \#2.

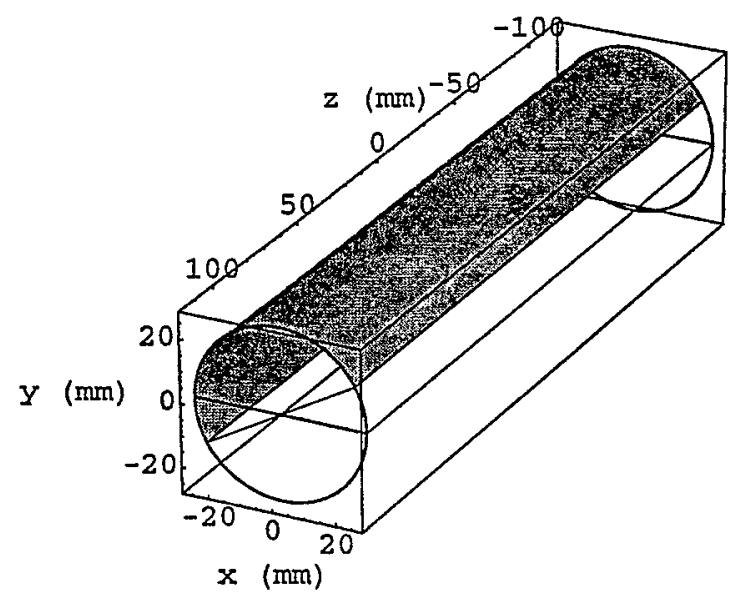

Fig.3 Dipole winding with opening angle $\pi$. 


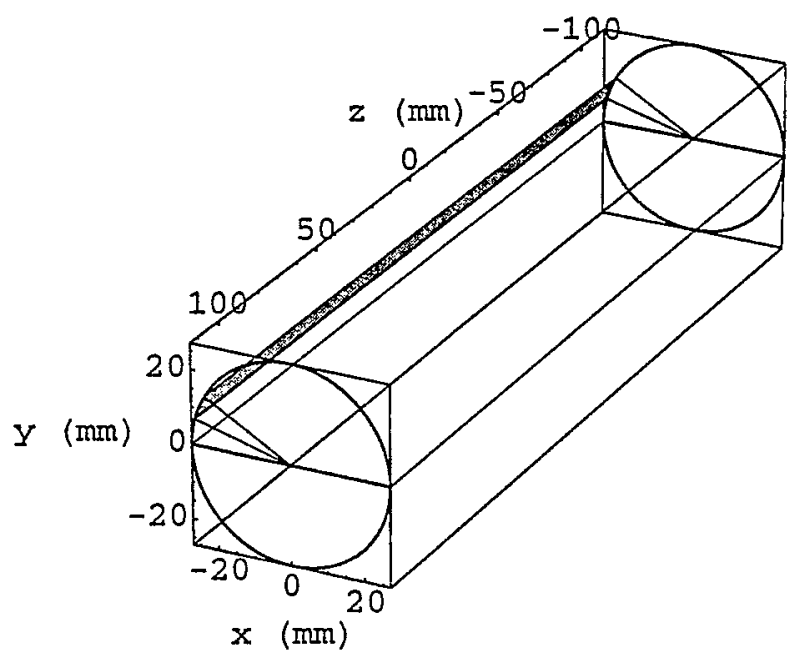

Fig.4 Tangential winding with opening angle $\pi / 12$ (15 degree).

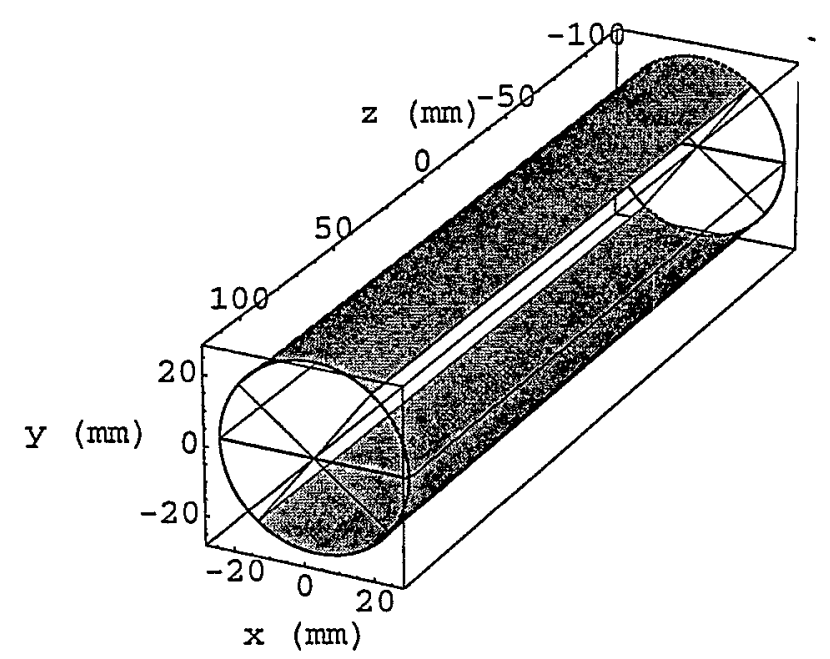

Fig.5 Quadrupole winding with the opening angle $\pi / 2 \times 2$. 
Table 1 Sensitivity factors for the helical magnetic flux enclosed by dipole, tangential, quadrupole windings.

$\begin{array}{lllll}\text { n } & \text { pole } & \text { Dipole } & \text { Tangential } & \text { Quadrupole } \\ 1 & \text { dipole } & 1 . & 1 . & 0 \\ 2 & \text { quadrupole } & 0 & 0.839 & 0.598 \\ 3 & \text { sextupole } & 0.231 & 0.677 & 0 \\ 4 & \text { octupole } & 0 & 0.524 & 0 \\ 5 & \text { decapole } & 0.083 & 0.387 & 0 \\ 6 & \text { dodecapole } & 0 & 0.27 & 0.0706 \\ 7 & 14 \text {-pole } & 0.0288 & 0.175 & 0 \\ 8 & 16 \text {-pole } & 0 & 0.102 & 0 \\ 9 & 18 \text {-pole } & 0.00684 & 0.0484 & 0 \\ 10 & 20 \text {-pole } & 0 & 0.0124 & 0.00238 \\ 11 & 22 \text {-pole } & 0.00124 & 0.00939 & 0 \\ 12 & 24 \text {-pole } & 0 & 0.0205 & 0 \\ 13 & 26 \text {-pole } & 0.00316 & 0.024 & 0 \\ 14 & 28 \text {-pole } & 0 & 0.0227 & 0.00433 \\ 15 & 30 \text {-pole } & 0.00266 & 0.0188 & 0\end{array}$
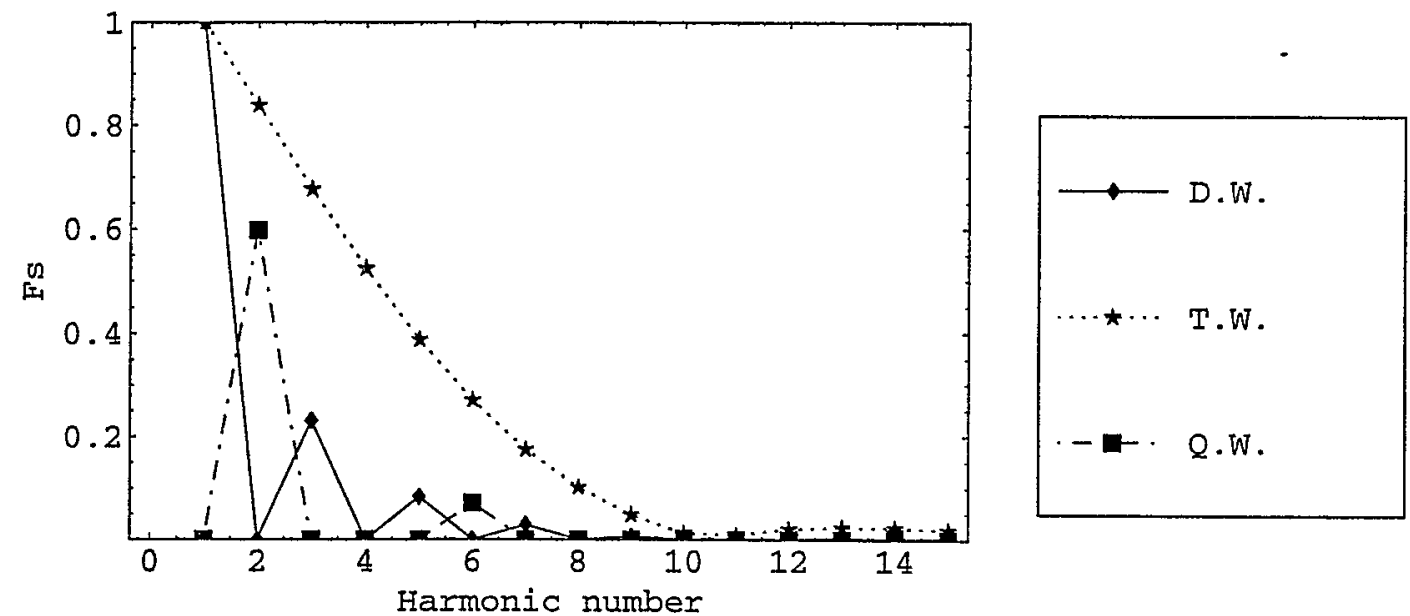

Fig.6 Harmonic dependence of sensitivity factors for the helical magnetic flux enclosed by dipole, tangential, quadrupole windings. 